\title{
Ezrin overexpression in gastrointestinal stromal tumors: an independent adverse prognosticator associated with the non-gastric location
}

\author{
Yu-Ching Wei ${ }^{1}$, Chien-Feng $\mathrm{Li}^{2}$, Shih-Chen $\mathrm{Yu}^{1}$, Fong-Fu Chou ${ }^{3}$, Fu-Min Fang ${ }^{4}$, \\ Hock-Liew Eng ${ }^{1}$, Yih-Huei Uen ${ }^{5}$, Yu-Fang Tian ${ }^{5}$, Jing-Mei Wu ${ }^{1}$, Shau-Hsuan Li ${ }^{6}$, \\ Wen-Wei Huang ${ }^{7}$, Wei-Ming Li $^{8}$ and Hsuan-Ying Huang ${ }^{1}$
}

\begin{abstract}
${ }^{1}$ Department of Pathology, Chang Gung Memorial Hospital-Kaohsiung Medical Center, Chang Gung University College of Medicine, Kaohsiung, Taiwan; ${ }^{2}$ Department of Pathology, Chi-Mei Foundation Medical Center, Tainan, Taiwan; ${ }^{3}$ Department of Surgery, Chang Gung Memorial Hospital-Kaohsiung Medical Center, Chang Gung University College of Medicine, Kaohsiung, Taiwan; ${ }^{4}$ Department of Radiation Oncology, Chang Gung Memorial HospitalKaohsiung Medical Center, Chang Gung University College of Medicine, Kaohsiung, Taiwan; ${ }^{5}$ Department of Surgery, Chi-Mei Foundation Medical Center, Tainan, Taiwan; ${ }^{6}$ Division of Oncology, Department of Internal Medicine, Chang Gung Memorial Hospital-Kaohsiung Medical Center, Chang Gung University College of Medicine, Kaohsiung, Taiwan; ${ }^{7}$ Department of Family Medicine, Buddhist Dalin Tzu Chi General Hospital, Chiayi, Taiwan and ${ }^{8}$ Department of Urology, Kaohsiung Medical University Hospital, Kaohsiung Medical University, Kaohsiung, Taiwan
\end{abstract}

\begin{abstract}
Ezrin, a member of the ezrin-radixin-moesin family, acts as a link between the cell membrane and actin cytoskeleton to integrate cell adhesion-mediated signaling. It implicates tumor progression, metastatic dissemination, and adverse outcomes in several cancer types, including pediatric and adult sarcomas. Although ezrin upregulation was shown by cDNA expression profiling, no study has systematically evaluated the significance of ezrin expression in a large cohort of gastrointestinal stromal tumors (GISTs). Ezrin immunostaining was carried out on tissue microarrays of primary GISTs and assessable in 347 cases, 188 of which were successfully evaluated for mutation variants of KIT and PDGFRA receptor tyrosine kinase (RTK) genes by sequencing with or without screening by denatured high-performance liquid chromatography. These GISTs with known RTK genotypes were dichotomized into two prognostically different groups. The endogenous expression and phosphorylation of ezrin in GIST cell lines were analyzed by western blotting. By immunohistochemistry, ezrin overexpression was present in $66 \%$ of GISTs and significantly associated with the nongastric location $(P=0.002)$ and decreased disease-free survival $(P=0.032$, univariately). However, it was not related to the National Institute of Health (NIH) risk category, Ki-67 labeling index, RTK genotypes, and other variables. In multivariate analyses, ezrin overexpression remained independently predictive of adverse outcome $(P=0.008$, risk ratio $=2.363)$, together with $\mathrm{Ki}-67$ labeling index $>5 \%(P<0.001$, risk ratio $=3.581)$, high-risk category $(P<0.001$, risk ratio $=2.156)$, and the non-gastric location $(P=0.029$, risk ratio $=1.899)$. Despite the variation in the ezrin expression level, phosphorylated ezrin at threonine ${ }^{567}$ was only detectable in GIST882 and GIST48 cells, but not in colonic smooth muscle cells. In conclusion, ezrin is frequently overexpressed in GISTs, especially those arising from the non-gastric sites. Given that its impact is independent of the NIH risk category, cell proliferation, and tumor location, ezrin immunoreactivity represents a valuable prognostic adjunct of GISTs, suggesting a causative role in conferring an aggressive phenotype. Modern Pathology (2009) 22, 1351-1360; doi:10.1038/modpathol.2009.107; published online 31 July 2009
\end{abstract}

Keywords: GIST; ezrin; RTKs; mutation; prognosis

Correspondence: Dr H-Y Huang, Department of Pathology, Chang Gung Memorial Hospital-Kaohsiung Medical Center, Chang Gung University College of Medicine, 123 Ta-Pei Road, Niao-Sung Township, Kaohsiung County 833, Taiwan.

E-mail: a120600310@yahoo.com

This work has been presented in part in the 98th annual meeting of the United States and Canadian Academy of Pathology Boston, USA, 7-13 March 2009).

Received 14 April 2009; revised 15 June 2009; accepted 16 June 2009; published online 31 July 2009
Gastrointestinal stromal tumors (GISTs) are the most common mesenchymal neoplasms of the gastrointestinal tract. ${ }^{1}$ On the basis of similarities in the immunohistochemical and ultrastructural features, it is believed that GISTs arise from interstitial cells of Cajal or their precursors. ${ }^{2}$ Approximately $75-90 \%$ of GISTs are characterized by activating mutations of the receptor tyrosine kinase (RTK) proto-oncogenes, mostly affecting KIT and rarely involving PDGFRA. ${ }^{3-5}$ 
The clinical behavior of GISTs is broadly varied and sometimes poses challenges in prognostication. Although the National Institute of Health (NIH) scheme has been proved to be prognostically useful, the implication of tumor location was not incorporated into this scheme. ${ }^{6-8}$ Moreover, it has become explicit that variations in the RTK gene mutations can affect tumor aggressiveness and response to targeted therapy with imatinib. ${ }^{9-12}$ Therefore, it is desirable to evaluate novel biomarkers with differential expression in cellular signaling pathways for developing prognostic adjuncts and/or alterative therapeutic strategies.

As a member of the ezrin-radixin-moesin (ERM) protein family, ezrin functions as a link between the plasma membrane and cortical actin cytoskeleton. ${ }^{13}$ Similar to other ERM proteins, the N-terminal FERM domain of ezrin is masked by binding of the C-terminal tail in inactive conformation through intermolecular and/or intramolecular associations. On stimuli transduced from the functional interaction of various growth factors and receptors, ezrin is activated to participate in signaling programs to modulate pleiotropic cellular functions, such as substrate adhesion, cell survival, cell migration, and formation of cell-cell junctions, and so on. ${ }^{14-16}$ There is mounting evidence that elevated ezrin expression through transcriptional upregulation accounts for the high metastatic propensity in a variety of epithelial and mesenchymal malignancies. ${ }^{17-22}$ Among the latter, ezrin overexpression is not only involved in the dissemination of pediatric rhabdomyosarcomas and osteosarcomas but is also highly associated with the adverse outcome in common adult soft-tissue sarcomas. ${ }^{17,19,22}$ More intriguingly, two recent studies using cDNA expression microarrays have identified ezrin as a frequently upregulated gene in GISTs and presumptively indicated its associations with distinct RTK mutation subtypes and tumor progression, respectively. ${ }^{23,24}$ However, the status and significance of ezrin expression have never been systematically assessed in a large, well-characterized cohort of human GIST specimens. By using tissue microarraybased immunohistochemistry, we therefore analyzed (i) the associations of ezrin expression with important prognostic variables of GIST, such as NIH risk category, proliferative index, and RTK genotypes, and also (ii) whether ezrin overexpression confers an independent prognostic implication. Furthermore, western blotting was applied to analyze the endogenous expression level and activating status of ezrin in GIST cell lines compared with primary human colonic smooth muscle cells.

\section{Materials and methods}

\section{Patients and Tumor Materials}

This retrospective study was carried out in accordance with the guidelines of the institutional review board (IRB98-0426B). From the archives of two tertiary medical centers, we identified primary localized GISTs of 372 consecutive patients, who had available tissue blocks, underwent surgical resection between January 1985 and December 2004, and did not receive imatinib treatment before disease relapses. All of these 372 cases were previously confirmed to be GISTs by immunohistochemistry and enrolled to construct tissue microarrays for ezrin immunostaining. ${ }^{25}$ The follow-up information, treatment course, and clinicopathological variables evaluated, including NIH risk category, were detailed in our previous studies. ${ }^{25-27}$

\section{Tissue Microarray-Based Immunohistochemistry for Ezrin}

The tissue microarray sections were prepared and heated as previously described for antigen retrieval, ${ }^{25}$ followed by incubation for $1 \mathrm{~h}$ with the primary antibody of ezrin (18, 1:50; BD Pharmingen, San Diego, CA, USA), and detection with the ChemMate EnVision kit (K5001; DAKO, Glostrup, Denmark). One pathologist (YCW) blinded to clinicopathological, genotyping, and outcome data independently assessed ezrin expression of GISTs included in tissue microarrays. The percentage of tumor cells with moderate or strong cytoplasmic immunoreactivity was recorded; only cases containing two or more preserved tissue cores were scored, and scores of multiple cores from the same patient were averaged to obtain a mean ezrin labeling index. The cutoff of the mean labeling index to define ezrin overexpression was cytoplasmic reactivity in $\geqslant 50 \%$ tumor cells (see statistical methods). Most tissue microarray blocks used for ezrin immunohistochemistry had also been previously sectioned and scored for Ki-67 staining in our earlier report, in which the mean labeling index $>5 \%$ was adopted as the cutoff to define Ki-67 overexpression. ${ }^{25}$

\section{Mutation Analysis of the RTK Genes}

The methods of DNA extraction, PCR amplification, direct sequencing of KIT exon 11, and screening by denatured high-performance liquid chromatography for exons 9, 13, and 17 of the KIT gene and exons 12 and 18 of the PDGFRA gene with confirmatory sequencing were as previously described. ${ }^{27}$

\section{Western Blotting Assays}

Equal amounts of total protein $(40 \mu \mathrm{g})$ extracted from GSIT48 and GIST882 cell lines (kind gifts of Dr Jonathan Fletcher) and primary human colonic smooth muscle cells (ScienCell Research Laboratories, Carlsbad, CA, USA) were separated on SDS$10 \%$ PAGE, transferred to PVDF membranes (Amersham Biosciences, Buckinghamshire, UK), 
and then blocked with 5\% skimmed milk in TBST buffer at room temperature for $1 \mathrm{~h}$. Afterward, the membranes were probed with antibodies at $4^{\circ} \mathrm{C}$ overnight against total ezrin (18, 1:250, BD Pharmingen), phosphorylated ezrin at the threonine ${ }^{567}$ ( $\mathrm{Thr}^{567}$ ) residue (J37, 1:250, BD Pharmingen), and GAPDH for a loading control (1:3000, MAB347, Chemicon) and then incubated with the secondary antibody at room temperature for $1.5 \mathrm{~h}$. Enhanced chemiluminescence reagents (Amersham Biosciences) were used to visualize the targeted proteins, which were then semi-quantitatively measured by densitometry.

\section{Follow-up and Statistical Analyses}

Statistical analyses were carried out using the SPSS 14 software package. Associations and comparisons of ezrin expression with various parameters were evaluated by $\chi^{2}$ or Student's $t$-test as appropriate for 347 cases with the interpretable ezrin labeling index. In this cohort, 313 patients had available follow-up data with a median duration of 57 months as of August 2007 (mean, 36.6; range, 1-235) forming the basis for univariate prognostic analysis. At last follow-up, 196 patients were alive without relapsed disease, 53 developed tumor relapses, including local recurrences in 29 and hepatic and/ or peritoneal dissemination in 40, 35 died of GISTs, and 23 died of unrelated causes. The end point analyzed was disease-free survival, which would not be confounded by imatinib therapy for patients with disseminated disease as seen in the evaluation of overall or disease-specific survival. A series of cutoff values in 5\% increment were tested for continuous variables, such as the ezrin labeling index, and those giving the best $P$-values were adopted to plot the Kaplan-Meier curves and compare prognostic differences by log-rank tests. RTK genotypes were dichotomized into two prognostically different groups as detailed in our previous report. ${ }^{27}$ The Kaplan-Meier curves were plotted for individual clinicopathological and molecular variables to compare the prognostic differences by log-rank tests. In the Cox multivariate regression model, all significant parameters identified at the univariate level were entered to compare the independent prognostic impact. However, as component factors of the NIH risk scheme, tumor size and mitotic activity were not introduced in multivariate comparisons. For all analyses, twosided tests of significance were used with $P<0.05$ considered significant.

\section{Results}

\section{Genotyping of RTK Genes}

In this study, mutation analysis of RTK genes was successfully carried out in 188 GISTs with muta- tions detected in 165 cases (88\%, Figure 1a-e). The trends of survival curves for individual RTK genotypes were generally in keeping with our earlier report and those published recently. ${ }^{27-29}$ These 188 cases were further dichotomized as favorable $(n=95)$ versus unfavorable $(n=93)$ genotypes according to our previous grouping rationale. ${ }^{27}$ The former group comprised (i) PDGFRA mutation involving exons 12 or 18 in nine cases (Figure 1b), (ii) 3' tandem insertion of KIT exon 11 with or without point mutation in 14 cases, and (iii) single point mutation of KIT exon 11 in 72 cases (Figure 1c). The group of unfavorable genotypes included (i) Ala502-Tyr503 insertion of KIT exon 9 in three cases (Figure 1d), (ii) wild type for both KIT and PDGFRA genes in 23 cases, and (3) $5^{\prime}$ deletion of KIT exon 11 with or without point mutation in 67 cases (Figure 1e). Of the KIT exon 11-deleted subgroup, there were 42 and 25 cases with deletions involving codons 557-558 and other codons, respectively. No mutation of KIT exon 13 or 17 was detected in this series.

\section{Immunohistochemical Expression of Ezrin and Its Correlations with Other Parameters in GISTs}

The immunohistochemical expression of ezrin could be successfully interpreted in 347 cases. This cohort consisted of 166 males and 181 females with a median age of 61 years. On the basis of the NIH consensus scheme, 119 cases were classified as very low or low risk (Figure 2a), 109 as intermediate risk (Figure 2b), and 115 as high risk (Figure 2c). However, the risk category of four remote cases could not be determined because of the lack of data of tumor size. As shown in Table 1, ezrin overexpression was present in 229 cases (66\%; Figure $2 \mathrm{~d}-\mathrm{f}$ ) and was significantly more frequent in GISTs affecting males $(P=0.023)$ and the non-gastric sites $(P=0.002)$. However, we could not substantiate the associations of ezrin overexpression with other clinicopathological factors, including the NIH risk category, Ki-67 labeling index, and RTK genotypes, in this cohort.

\section{Phosphorylation of Ezrin at the Thr ${ }^{567}$ Residue was Detectable Only in the GIST Cell Lines but not in the Primary Cells of Human Colonic Smooth Muscle}

To modulate the cytoskeletal rearrangements, the $\mathrm{Thr}^{567}$ residue at the C-terminal domain of ezrin must be activated by phosphorylation to convert ezrin from a dormant cytoplasmic form to a membrane- and actin-binding conformation. ${ }^{13}$ Accordingly, we further compared the endogenous expression and activation status of ezrin in GIST cell lines versus HISMC cells. By western blotting, the ezrin expression of GIST882 cells was almost as abundant as that of human colonic smooth muscle 


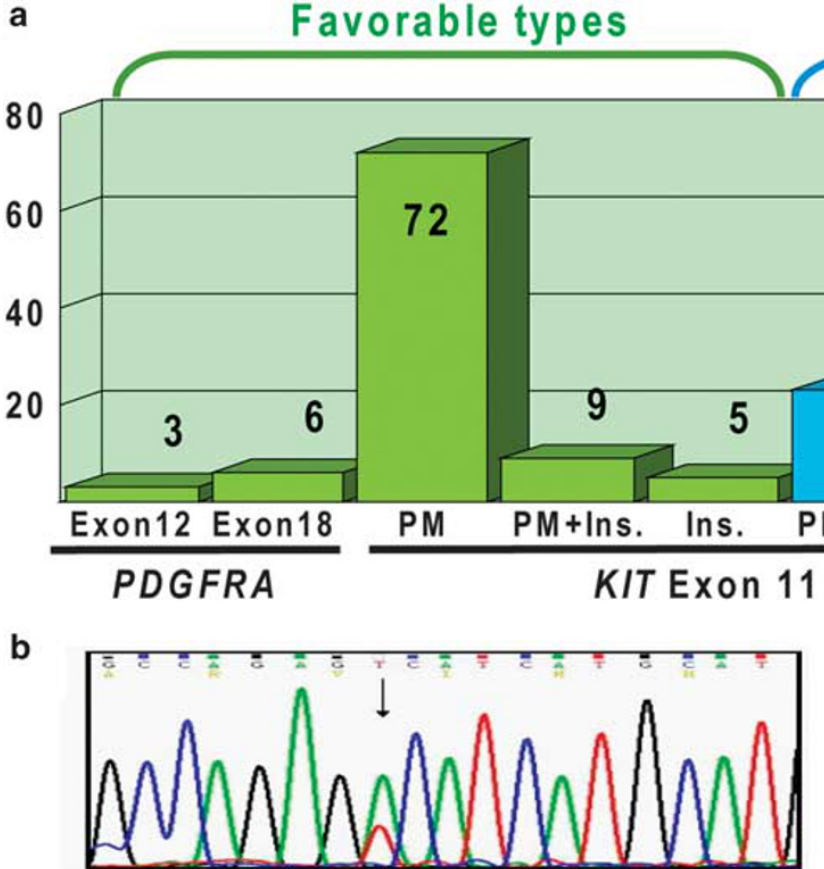

Point mutation of D842V in PDGFRA exon 18

d

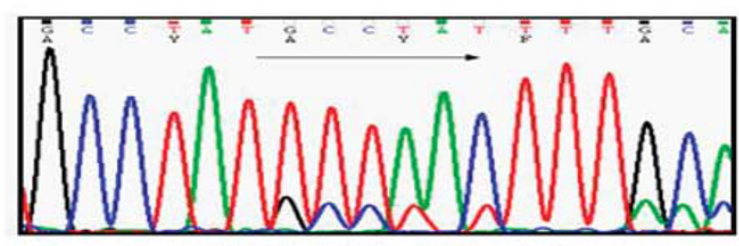

Insertion of A502-Y503 in KIT exon 9
Unfavorable types
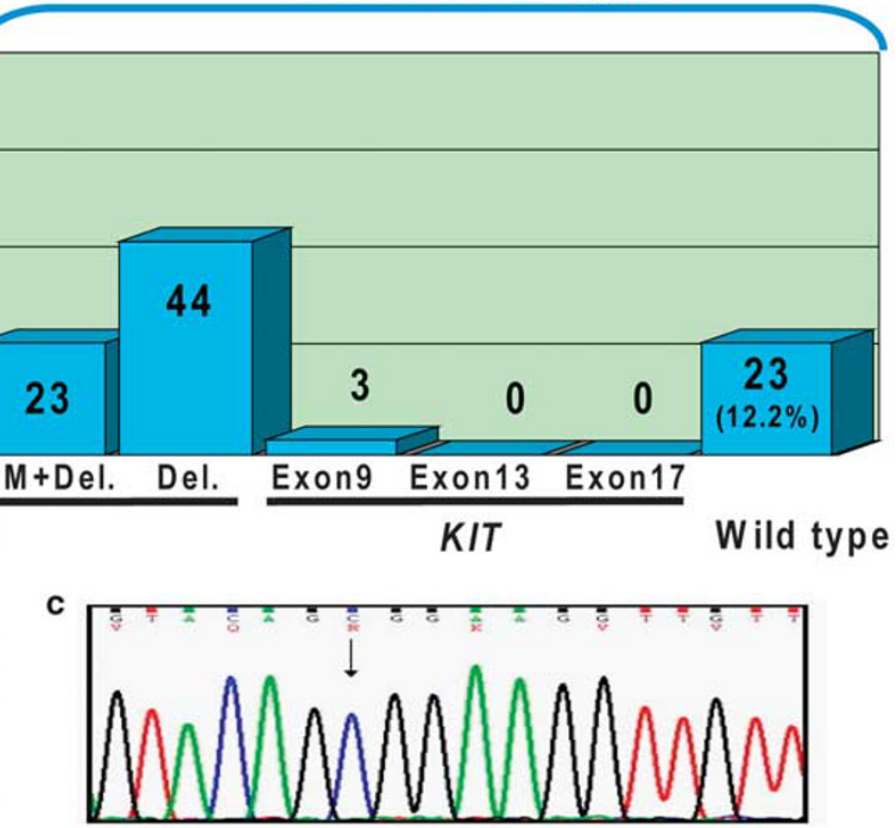

Point mutation of W557R in KIT exon 11

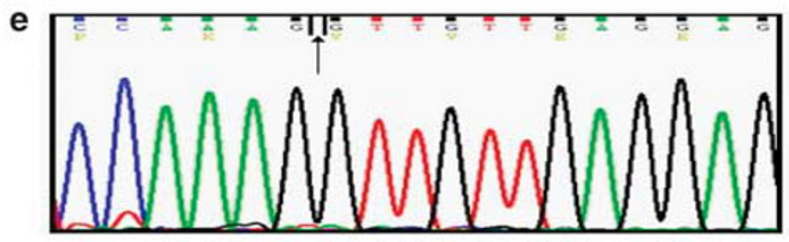

Deletion of P551-K558 in KIT exon 11

Figure 1 Mutation analysis and dichotomous classification of receptor tyrosine kinase (RTK) genotypes in 188 GISTs with informative data. (a) A histogram shows the frequency distribution of various KIT or PDGFRA mutation types. The most common mutation subtype was single point mutations in KIT exon 11, followed by deletions in KIT exon 11 without point mutations. Note that 23 (12\%) cases showed wild genotypes for both KIT and PDGFRA genes. Two prognostically different groups were identified and referred to as favorable (green) and unfavorable (blue) genotypes. Sequencing showed nucleotide alterations in representative GISTs with favorable ((b), point mutation of D842 V in PDGFRA exon 18; (c), point mutation of W557R in KIT exon 11) and unfavorable ((d), insertion of A502-Y503 in KIT exon 9; (e), deletion of P551-K558 in KIT exon 11) genotypes of RTKs. PM: point mutation; DEL: deletion; INS: insertion.

cells, whereas it was comparatively lower in GIST 48 cells (Figure 3, row 1). Interestingly, phosphorylated ezrin at $\mathrm{Thr}^{567}$ was only detectable in GIST cell lines but not in smooth muscle cells, and the expression level was higher in GIST882 cells than in GIST48 cells (Figure 3, row 2). The latter finding of phosphorylated ezrin expression was essentially reflective of what was obtained by probing total ezrin. However, it must be added that the monoclonal phospho-ezrin antibody used has been reported to partially cross-react with two other members of the ERM protein family, for example, radixin and moesin, on phosphorylated $\mathrm{Thr}^{564}$ and $\mathrm{Thr}^{558}$ residues, respectively.

\section{Survival Analyses}

Correlations of clinical outcomes with various clinicopathological, immunohistochemical, and mole- cular parameters at the univariate level are shown in Table 2 and Figure 4. In this series, similar to our and others' previous reports, inferior disease-free survival was significantly associated with several clinicopathological factors, including older age $(P=0.048)$, non-gastric location $(P=0.035)$, presence of epithelioid histology $(P<0.001)$, larger tumor size $(P<0.001)$, higher mitotic count $(P<0.001)$, and increasing NIH risk levels $\left(P<0.001\right.$, Figure 4a).$^{5,6,9,13,30-33}$ In addition, high proliferative index $(P<0.001$, Figure $4 \mathrm{~b})$ and unfavorable RTK genotypes $(P<0.001$, Figure 4c) were both highly predictive of adverse outcomes. It is noted that ezrin overexpression was also significantly associated with inferior disease-free survival $(P=0.032$, Figure $4 \mathrm{~d})$.

In multivariate analysis (Table 3), ezrin overexpression remained prognostically independent $(P=0.008)$, along with high NIH risk level $(P<0.001)$, high Ki-67 index $(P<0.001)$, and 
a
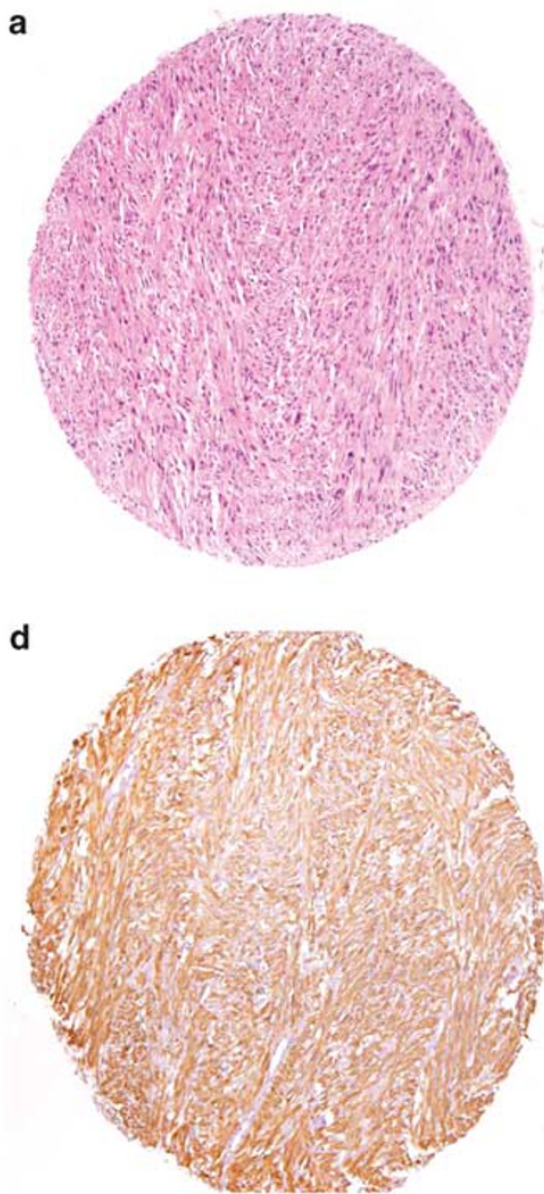

b

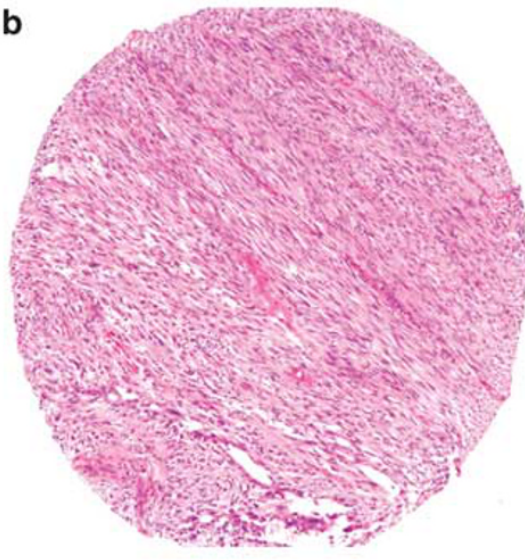

e

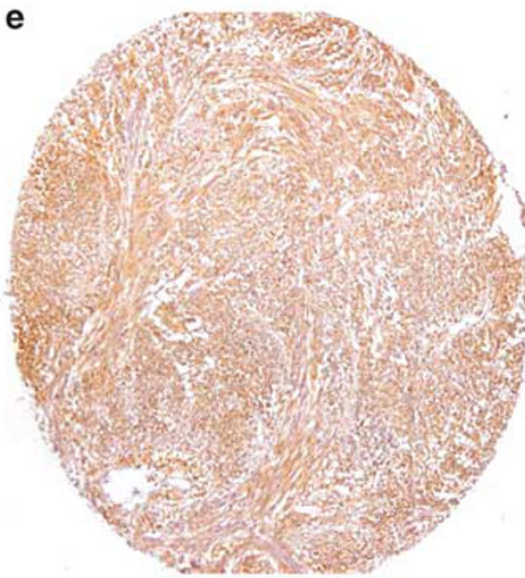

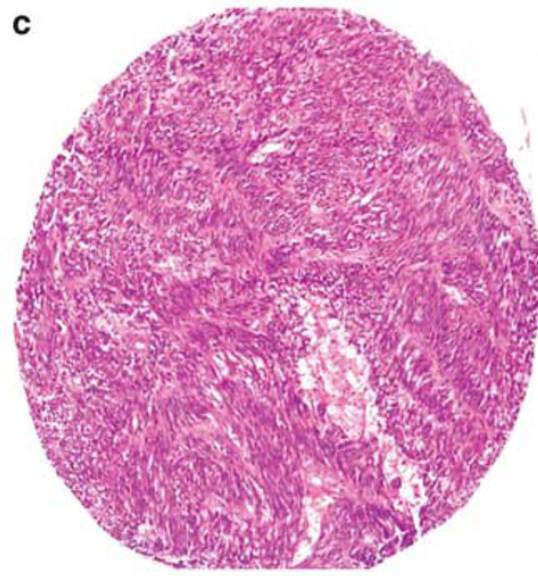

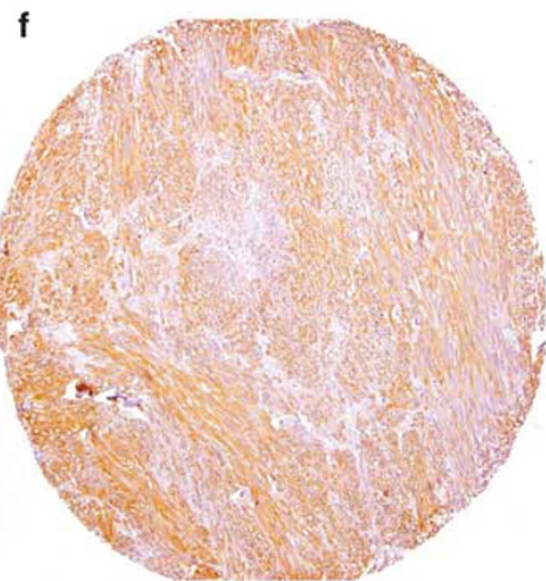

Figure 2 Representative examples of GIST tissue microarray, stained with hematoxylin and eosin and ezrin. Photomicrographs of GISTs classified as (a) low risk, (b) intermediate risk, and (c) high risk according to the NIH consensus criteria with increasing mitoses. Ezrin immunostaining shows no apparent difference in expression levels in each representative case of (d) low-risk, (e) intermediate-risk, and (f) high-risk GISTs.

non-gastric location $(P=0.029)$. Moreover, ezrin overexpression also identified patients at a more than twofold higher risk of relapsed disease. However, mutation genotypes, old age, and the presence of epithelioid histology lost statistical significance.

\section{Discussion}

On the basis of cDNA expression profiling, two different groups recently showed that the ezrin/VIL2 gene was differentially expressed among GISTs with validation by either quantitative RT-PCR or western blotting assay. ${ }^{23,24}$ Of these, Koon et $a l^{23}$ found that the expression level of ezrin was significantly higher in malignant GISTs than its counterparts with benign or uncertain malignant behavior. In the series of Subramanian et $\mathrm{al}^{24}$, ezrin was preferentially overexpressed in a subset of GISTs with KIT mutation in either exon 11 or exon 9, despite that its low expression was still at the detectable level in GISTs with PDGFRA mutation. In this study, we have first systematically evaluated the frequency and prognostic relevance of ezrin overexpression and its correlations with clinicopathological and molecular variables in a large cohort of human GIST specimens. Our findings showed that ezrin overexpression, present in approximately two-thirds of GISTs, had a modest correlation with gender. Except for tumor localization, we could not substantiate the correlations of ezrin overexpression with other important variables related to tumor aggressiveness, including NIH risk category and RTK genotypes. Nevertheless, it was noteworthy that ezrin overexpression was identified as an independent poor prognosticator of disease-free survival with a more than twofold increased risk of tumor relapse.

It has become more evident that a high expression of ezrin promotes disease progression and metastasis in a variety of primary pediatric and adult cancers, thereby conferring poorer patient survival. ${ }^{17-22}$ Although this adverse prognostic effect has been established in human malignant neoplasms derived from both epithelial and mesenchymal origins, exceptions to this general rule were occasionally reported, suggesting that there may be cellspecific functions for ezrin in tumor progression. ${ }^{17-22}$ For instance, the loss of ezrin expression in serous 
Table 1 Associations of ezrin expression with various clinicopathological parameters

\begin{tabular}{|c|c|c|c|c|}
\hline & Number & $\begin{array}{c}\text { Low } \\
\text { expression }\end{array}$ & $\begin{array}{c}\text { Over- } \\
\text { expression }\end{array}$ & $\mathrm{P}$-value \\
\hline \multicolumn{5}{|l|}{ Sex } \\
\hline Male & 166 & 46 & 120 & 0.023* \\
\hline Female & 181 & 72 & 109 & \\
\hline Age (years) & & $58.72 \pm 12.174$ & $60.03 \pm 13.173$ & 0.371 \\
\hline \multicolumn{5}{|l|}{ Location } \\
\hline Gastric & 201 & 81 & 120 & 0.002* \\
\hline Non-gastric & 146 & 36 & 109 & \\
\hline \multicolumn{5}{|l|}{ Histological type } \\
\hline Spindle & 267 & 85 & 182 & 0.119 \\
\hline $\begin{array}{l}\text { Epithelioid } \\
\text { and mixed }\end{array}$ & 80 & 33 & 47 & \\
\hline Tumor size $(\mathrm{cm})$ & & $6.352 \pm 4.207$ & $6.630 \pm 4.491$ & 0.577 \\
\hline $\begin{array}{l}\text { Mitotic count } \\
\text { (50 HPFs) }\end{array}$ & & $10.03 \pm 28.799$ & $8.88 \pm 20.505$ & 0.670 \\
\hline \multicolumn{5}{|l|}{ NIH risk level } \\
\hline Low/very low & 119 & 39 & 80 & 0.338 \\
\hline Intermediate & 109 & 43 & 66 & \\
\hline High & 115 & 35 & 80 & \\
\hline $\begin{array}{l}\text { Ki-67 labeling } \\
\text { index }\end{array}$ & & $5.05 \pm 7.800$ & $5.23 \pm 6.657$ & 0.829 \\
\hline \multicolumn{5}{|l|}{ Mutation type } \\
\hline Favorable type & 95 & 32 & 63 & 0.395 \\
\hline $\begin{array}{l}\text { Unfavorable } \\
\text { type }\end{array}$ & 93 & 26 & 67 & \\
\hline
\end{tabular}

HPFs: high power fields.

\section{GIST48 GIST882 HCSMC}

\section{Ezrin}

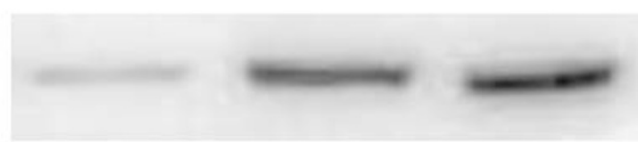

pEzrin $^{\text {Thr567 }}$

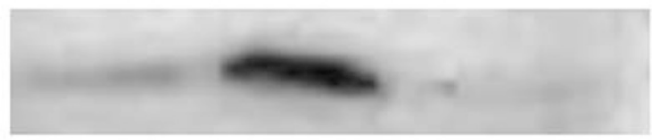

\section{GAPDH}

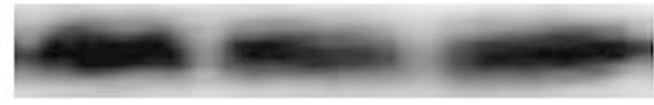

Figure 3 Western blotting assays to detect expression of total ezrin and phosphorylated ezrin at $\mathrm{Thr}^{567}$ in GIST cell lines and primary human colonic smooth muscle cells. Detection of total ezrin shows similarly abundant expression in GIST882 and HISCM cells, whereas GIST 48 cells have a comparatively lower expression level (row 1). Phosphorylated ezrin at $\mathrm{Thr}^{567}$ is only detectable in the cells of GIST but not in human colonic smooth muscle cells, with the expression level being higher in GIST882 than in GIST48 (row 2). GAPDH serves as the loading control (row 3). HCSMC, human colonic smooth muscle cells.

ovarian carcinomas was shown to be associated with poor survival. ${ }^{34}$ Moreover, unlike osteosarcomas and adult high-grade sarcomas, ezrin over-
Table 2 Univariate log-rank analyses for ezrin expression status and other prognostic factors with respect to disease-free survival

\begin{tabular}{|c|c|c|c|c|}
\hline \multirow[b]{2}{*}{ Parameters } & \multirow[b]{2}{*}{ Category } & \multirow[b]{2}{*}{$\begin{array}{l}\text { No. of } \\
\text { case }\end{array}$} & \multicolumn{2}{|c|}{ Disease-free survival } \\
\hline & & & $\begin{array}{l}\text { No. of } \\
\text { event }\end{array}$ & $\mathrm{P}$-value \\
\hline \multirow[t]{2}{*}{ Sex } & Male & 151 & 43 & 0.877 \\
\hline & Female & 162 & 49 & \\
\hline \multirow[t]{2}{*}{ Age (years) } & $<70$ & 230 & 63 & $0.048^{*}$ \\
\hline & $\geq 70$ & 82 & 29 & \\
\hline \multirow{2}{*}{ Location } & Gastric & 183 & 44 & $0.035^{*}$ \\
\hline & Non-gastric & 130 & 48 & \\
\hline \multirow[t]{2}{*}{ Histological type } & Spindle & 240 & 57 & $<0.001^{*}$ \\
\hline & Mixed/epithelioid & 73 & 35 & \\
\hline \multirow[t]{3}{*}{ Tumor size $(\mathrm{cm})$} & $\leq 5 \mathrm{~cm}$ & 141 & 25 & $<0.001 *$ \\
\hline & $>5 ; \leq 10 \mathrm{~cm}$ & 120 & 37 & \\
\hline & $>10 \mathrm{~cm}$ & 51 & 29 & \\
\hline \multirow{3}{*}{$\begin{array}{l}\text { Mitotic count } \\
\text { (50 HPFs) }\end{array}$} & $0-5$ & 223 & 43 & $<0.001 *$ \\
\hline & $6-10$ & 40 & 16 & \\
\hline & $>10$ & 48 & 33 & \\
\hline \multirow[t]{3}{*}{ NIH risk level } & Very low/low & 112 & 16 & $<0.001$ * \\
\hline & Intermediate & 99 & 18 & \\
\hline & High & 101 & 58 & \\
\hline \multirow[t]{2}{*}{ Mutation type } & Favorable type & 94 & 18 & $<\mathbf{0 . 0 0 1}^{*}$ \\
\hline & Unfavorable type & 90 & 46 & \\
\hline \multirow{2}{*}{$\begin{array}{l}\text { Ki-67 labeling } \\
\text { index }\end{array}$} & $\leq 5$ & 212 & 48 & $<0.001 *$ \\
\hline & $>5$ & 83 & 39 & \\
\hline \multirow[t]{2}{*}{ Ezrin } & Low expression & 101 & 24 & $0.032^{*}$ \\
\hline & Overexpression & 212 & 68 & \\
\hline
\end{tabular}

expression was recently found not predictive of clinical outcomes in synovial sarcoma. ${ }^{35}$ In our series, the high proportion and independent prognostic value of ezrin overexpression in GISTs suggest that this membrane/cytoskeleton anchor within the ERM protein family may represent a potential prognostic adjunct and/or therapeutic target and that those patients with ezrin-overexpressing GISTs should be closely monitored postoperatively.

Previous studies on GISTs have shown that anatomic sites correlate with the variations in histomorphology, clinical outcome, and RTK genotypes. ${ }^{7,8,12,28}$ First, gastric GISTs are generally known to follow a more favorable course than small intestinal GISTs. Second, GISTs with a $3^{\prime}$ end insertion of KIT exon 11 or with PDGFRA mutations (favorable RTK genotypes in this series) are known to preferentially arise from the stomach, whereas KIT exon 9 mutation (one variant of unfavorable RTK genotypes) affects the small bowel exclusively. ${ }^{12}$ Another finding of interest in this series was an intimate association of ezrin overexpression with non-gastric location, which was also an independent adverse prognosticator. In the study of Subramanian et $a l^{24}$, it remained elusive whether the comparatively lower ezrin expression in GISTs with PDGFRA mutation was simply because this particular genotypic subtype preferentially involves gastric GISTs. It is noted that Antonescu et $a l^{36}$ 

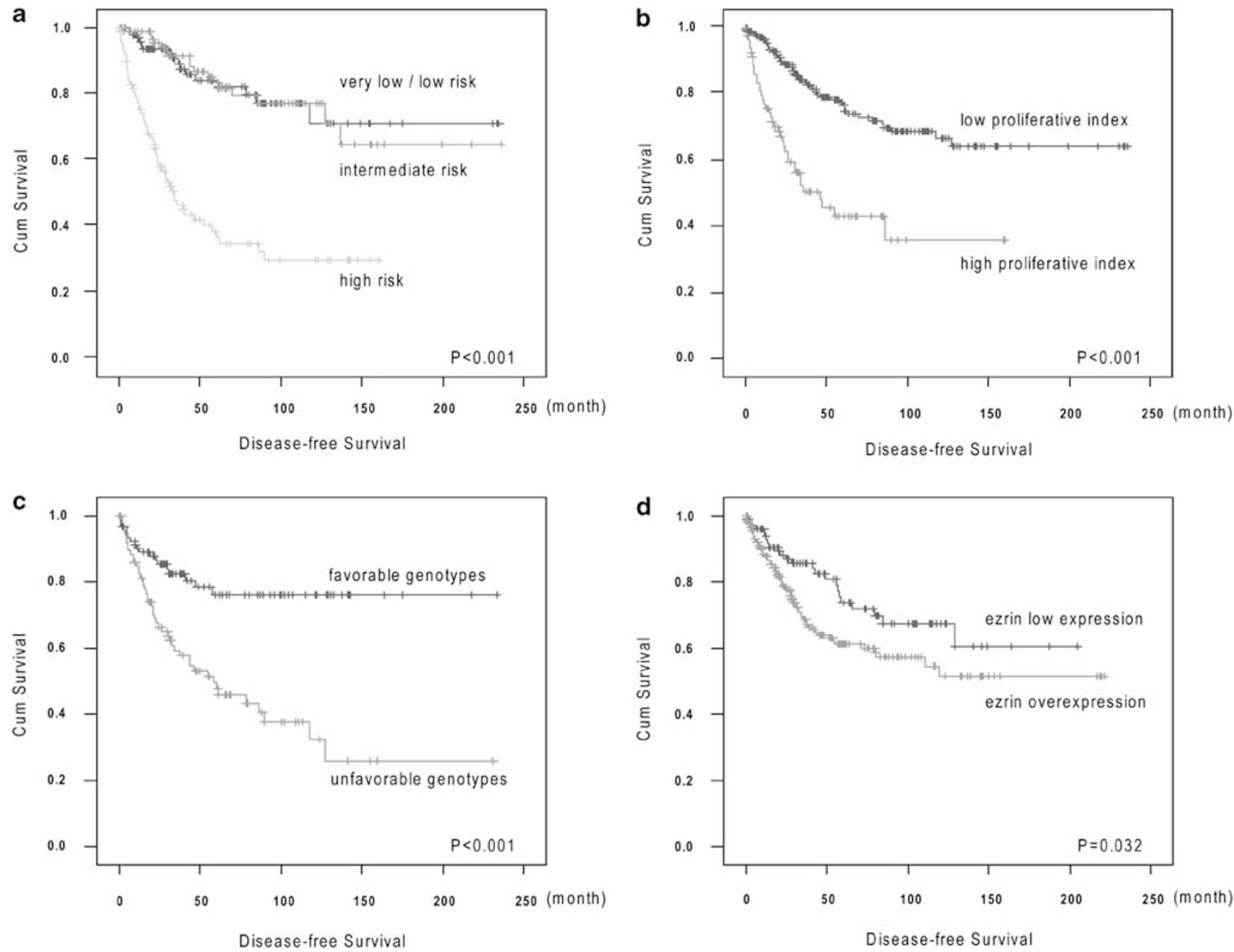

Figure 4 The Kaplan-Meier survival curves are plotted to predict disease-free survival according to the (a) NIH risk categories, (b) Ki-67 labeling index, (c) genotypes of KIT and PDGFRA genes, and (d) expression level of ezrin.

Table 3 Results of Cox multivariate analyses in relation to disease-free survival

\begin{tabular}{|c|c|c|c|c|}
\hline Parameter & Category & Risk ratio & $95 \% C I$ & P-value \\
\hline \multirow[t]{2}{*}{ Ki-67 labeling index } & $\leq 5$ & 1 & - & $\boldsymbol{P}<\mathbf{0 . 0 0 1}$ * \\
\hline & $>5$ & 3.581 & $1.995-6.426$ & \\
\hline \multirow[t]{2}{*}{ Ezrin } & Low expression & 1 & - & $\boldsymbol{P}=\mathbf{0 . 0 0 8}$ * \\
\hline & Overexpression & 2.363 & $1.254-4.454$ & \\
\hline \multirow[t]{2}{*}{ NIH risk level } & Very low/low/intermediate & 1 & - & $\boldsymbol{P}<\mathbf{0 . 0 0 1}$ * \\
\hline & High & 2.156 & $1.439-3.230$ & \\
\hline \multirow[t]{2}{*}{ Location } & Gastric & 1 & - & $P=0.029^{*}$ \\
\hline & Non-gastric & 1.899 & $1.068-3.377$ & \\
\hline \multirow[t]{2}{*}{ Mutation type } & Favorable & 1 & - & $P=0.092$ \\
\hline & Unfavorable & 1.657 & $0.920-2.981$ & \\
\hline \multirow[t]{2}{*}{ Histological type } & Spindle & 1 & - & $P=0.231$ \\
\hline & Mixed/epithelioid & 1.432 & $0.795-2.580$ & \\
\hline \multirow[t]{2}{*}{ Age } & $<70$ & 1 & - & $P=0.259$ \\
\hline & $\geq 70$ & 1.408 & $0.777-2.550$ & \\
\hline
\end{tabular}

recently showed that different functional genomic clusters could be distinguished by the anatomic sites of GISTs using oligonucleotide microarray analysis. Along these lines, future studies may be warranted to examine whether these site-dependent differentially expressed genes are functionally different in upregulating ezrin expression.

Currently, mutation variants of RTK genes have become understood to have a more distinctive role in the prognostication of GISTs. In this regard, this 
series was in accordance with others series, and our previous findings that GISTs with KIT exon 11 deletions, especially involving codons 557-558, show a more aggressive clinical course. ${ }^{12,28,29} \mathrm{~Pa}$ tients with KIT exon 11-deleted GISTs had the shortest disease-free survival at least in univariate analysis, compared with those with other RTK genotypic subtypes. Recently, Haller et $a l^{37}$ has partly elucidated the functional basis for this difference in clinical behavior by showing that GISTs with KIT exon 11 deletions are characterized by the hyperactivation and/or overexpression of ERK1/2, STAT3, mTOR, and EIF4E. Increased activation from these signaling intermediates may in turn converge to upregulate cyclin D with consequent hyperphosporylation of $\mathrm{RB}$ protein, thereby culminating in accelerated cell proliferation. In this study, we found no significant association of ezrin expression with the Ki-67 proliferative index and RTK genotypes, which was essentially in line with the previous reports showing that ezrin generally lacks a direct role in regulating cell proliferation. $^{38,39}$

Through the modulation of diverse actin-based functions, ezrin is known as a key component of the molecular chain connecting the metastasis-associated cell surface proteins with the signal transduction network. ${ }^{17-22}$ What remain less clear for this pathogenetic role of ezrin are the crucial elements of its various downstream effectors, which may vary among different tumor types. In the murine model of rhabdomyosarcoma, the prometastatic activity of ezrin was recently substantiated to be mediated in part through the Rho family of small $G$ proteins, consistent with the firmly established reciprocal relationship between the ERM proteins and Rho activity. ${ }^{17}$ However, activation of the p44/42 MAPK pathway is important for ezrin in preventing the death of tumor cells early in the process of metastasis to the lung in the murine model of osteosarcoma. ${ }^{19}$ In contrast, the activity of ezrin in Ewing's family of tumors is dependent on the AKT/mTOR signaling cascade rather than p44/42 MAPK. ${ }^{40}$ To date, there has been no study directly documenting the effector proteins of ezrin in GISTs. However, several lines of circumstantial evidence suggest that a potential candidate may be focal adhesion kinase (FAK), a cytoplasmic non-receptor kinase implicating the cytoskeletal dynamics to regulate cell motility. First, Koon et $a l^{23}$ showed that the transcripts of ezrin and FAK were frequently co-upregulated in malignant GISTs in a cDNA microarray study. Second, FAK can act as an upstream modulator to regulate the activity of AKT, a critical signaling kinase well recognized to enhance cell survival in GISTs. ${ }^{41}$ More intriguingly, FAK has recently been shown in cell line models to directly interact with ezrin to trigger its phosphorylation in a cell-matrix adhesion-independent manner. ${ }^{42}$

Ezrin is also known to integrate various oncogenic signals for cytoskeletal rearrangements through interaction with multiple classes of transmembrane receptors, including various RTKs. ${ }^{40}$ Among the latter, the epidermal growth factor receptor and c-Met can phosphorylate the N-terminal tyrosines of ezrin to promote cytoskeletal reorganization and consequent morphogenetic alterations. ${ }^{17}$ However, the question remains as to the upstream mechanisms of ezrin activation in GISTs. By western blotting, we found that only GIST cell lines, rather than human colonic smooth muscle cells, contained phosphorylated ezrin at $\mathrm{Thr}^{567}$, the hallmark of ezrin activation. Our finding also suggested that ezrin activity was essentially in proportion to the expression level of total ezrin in GISTs. More relevantly, ezrin has been identified as an essential downstream target of oncogenic Kit mutants in leukemic progression of murine proerythroblasts. ${ }^{43}$ In this context, it would appear tempting to examine in future studies whether this activation of ezrin by Kit mutants also holds true in human GISTs.

In conclusion, ezrin overexpression is present in approximately two-thirds of GISTs, especially in those arising from the non-gastric location. Along with the NIH risk category, Ki-67 proliferative index, and tumor location, ezrin overexpression is also an independent adverse prognosticator of disease-free survival, suggesting a causative role in conferring an aggressive phenotype. Our finding of ezrin immunoreactivity as a valuable prognostic adjunct of GISTs may justify a close post-operative follow-up for those cases with ezrin overexpression.

\section{Acknowledgements}

We are very grateful to Dr Jonathan Fletcher for kindly providing GIST 882 and GIST 48 cell lines. This work was supported in part by grants from the National Science Council, Taiwan (NSC 95-2320-B182A-007 -MY2) and Chang Gung Memorial Hospital (CMRPG850442, CMRPG880941, CMRPG880251), as well as the Chi-Mei Medical Center (CMFHR 9634).

\section{Disclosure/conflict of interest}

The authors declare no conflict of interest.

\section{References}

1 van der Zwan SM, DeMatteo RP. Gastrointestinal stromal tumor: 5 years later. Cancer 2005;104:1781-1788.

2 Kindblom LG, Remotti HE, Aldenborg F, et al. Gastrointestinal pacemaker cell tumor (GIPACT): gastrointestinal stromal tumors show phenotypic characteristics of the interstitial cells of Cajal. Am J Pathol 1998;152: 1259-1269.

3 Heinrich MC, Rubin BP, Longley BJ, et al. Biology and genetic aspects of gastrointestinal stromal tumors: KIT activation and cytogenetic alterations. Hum Pathol 2002;33:484-495. 
4 Corless CL, Fletcher JA, Heinrich MC. Biology of gastrointestinal stromal tumors. J Clin Oncol 2004; 22:3813-3825.

5 Heinrich MC, Corless CL, Duensing A, et al. PDGFRA activating mutations in gastrointestinal stromal tumors. Science 2003;299:708-710.

6 Miettinen M, El-Rifai W, Sobin LHL, et al. Evaluation of malignancy and prognosis of gastrointestinal stromal tumors: a review. Hum Pathol 2002;33:478-483.

7 Miettinen M, Sobin LH, Lasota J. Gastrointestinal stromal tumors of the stomach: a clinicopathologic, immunohistochemical, and molecular genetic study of 1765 cases with long-term follow-up. Am J Surg Pathol 2005;29:52-68.

8 Miettinen M, Makhlouf H, Sobin LH, et al. Gastrointestinal stromal tumors of the jejunum and ileum: a clinicopathologic, immunohistochemical, and molecular genetic study of 906 cases before imatinib with longterm follow-up. Am J Surg Pathol 2006;30:477-489.

9 Heinrich MC, Corless CL, Demetri GD, et al. Kinase mutations and imatinib response in patients with metastatic gastrointestinal stromal tumor. J Clin Oncol 2003;21:4342-4349.

10 Demetri GD, von Mehren M, Blanke CD, et al. Efficacy and safety of imatinib mesylate in advanced gastrointestinal stromal tumors. $\mathrm{N}$ Engl J Med 2002;347:472-480.

11 Verweij J, Casali PG, Zalcberg J, et al. Progression-free survival in gastrointestinal stromal tumours with highdose imatinib: randomised trial. Lancet 2004;364: 1127-1134.

12 Lasota J, Miettinen M. Clinical significance of oncogenic KIT and PDGFRA mutations in gastrointestinal stromal tumours. Histopathology 2008;53: 245-266.

13 Bretscher A, Edwards K, Fehon RG. ERM proteins and merlin: integrators at the cell cortex. Nat Rev Mol Cell Biol 2002;3:586-599.

14 Hunter KW. Ezrin, a key component in tumor metastasis. Trends Mol Med 2004;10:201-204.

15 Martin TA, Harrison G, Mansel RE, et al. The role of the CD44/ezrin complex in cancer metastasis. Crit Rev Oncol Hematol 2003;46:165-186.

16 Dransfield DT, Bradford AJ, Smith J, et al. Ezrin is a cyclic AMP-dependent protein kinase anchoring protein. EMBO J 1997;16:35-43.

$17 \mathrm{Yu} \mathrm{Y,} \mathrm{Khan} \mathrm{J,} \mathrm{Khanna} \mathrm{C,} \mathrm{et} \mathrm{al.} \mathrm{Expression} \mathrm{profiling}$ identifies the cytoskeletal organizer ezrin and the developmental homeoprotein Six-1 as key metastatic regulators. Nat Med 2004;10:175-181.

18 Ohtani K, Sakamoto H, Rutherford T, et al. Ezrin, a membrane-cytoskeletal linking protein, is involved in the process of invasion of endometrial cancer cells. Cancer Lett 1999;147:31-38.

19 Khanna C, Wan X, Bose S, et al. The membranecytoskeleton linker ezrin is necessary for osteosarcoma metastasis. Nat Med 2004;10:182-186.

20 Geiger KD, Stoldt P, Schlote W, et al. Ezrin immunoreactivity is associated with increasing malignancy of astrocytic tumors but is absent in oligodendrogliomas. Am J Pathol 2000;157:1785-1793.

$21 \mathrm{Kim}$ MS, Cho WH, Song WS, et al. Prognostic significance of ezrin expression in pleomorphic malignant fibrous histiocytoma. Anticancer Res 2007;27: 1171-1178.

22 Weng WH, Ahlen J, Astrom K, et al. Prognostic impact of immunohistochemical expression of ezrin in highly malignant soft tissue sarcomas. Clin Cancer Res 2005;11:6198-6204.

23 Koon N, Schneider-Stock R, Sarlomo-Rikala M, et al. Molecular targets for tumour progression in gastrointestinal stromal tumours. Gut 2004;53:235-240.

24 Subramanian S, West RB, Corless CL, et al. Gastrointestinal stromal tumors (GISTs) with KIT and PDGFRA mutations have distinct gene expression profiles. Oncogene 2004;23:7780-7790.

25 Huang HY, Huang WW, Lin CN, et al. Immunohistochemical expression of p16INK4A, Ki-67, and Mcm2 proteins in gastrointestinal stromal tumors: prognostic implications and correlations with risk stratification of $\mathrm{NIH}$ consensus criteria. Ann Surg Oncol 2006;13:1633-1644.

26 Huang HY, Li CF, Huang WW, et al. A modification of $\mathrm{NIH}$ consensus criteria to better distinguish the highly lethal subset of primary localized gastrointestinal stromal tumors: a subdivision of the original high-risk group on the basis of outcome. Surgery 2007;141:748-756.

27 Li CF, Huang WW, Wu JM, et al. Heat shock protein 90 overexpression independently predicts inferior diseasefree survival with differential expression of the alpha and beta isoforms in gastrointestinal stromal tumors. Clin Cancer Res 2008;14:7822-7831.

28 Martin J, Poveda A, Llombart-Bosch A, et al. Deletions affecting codons 557-558 of the c-KIT gene indicate a poor prognosis in patients with completely resected gastrointestinal stromal tumors: a study by the Spanish Group for Sarcoma Research (GEIS). J Clin Oncol 2005;23:6190-6198.

29 Andersson J, Bumming P, Meis-Kindblom JM, et al. Gastrointestinal stromal tumors with KIT exon 11 deletions are associated with poor prognosis. Gastroenterology 2006;130:1573-1581.

30 Fletcher CD, Berman JJ, Corless C, et al. Diagnosis of gastrointestinal stromal tumors: a consensus approach. Hum Pathol 2002;33:459-465.

31 Dematteo RP, Gold JS, Saran L, et al. Tumor mitotic rate, size, and location independently predict recurrence after resection of primary gastrointestinal stromal tumor (GIST). Cancer 2008;112:608-615.

32 Heinrich MC, Corless CL. Gastric GI stromal tumors (GISTs): the role of surgery in the era of targeted therapy. J Surg Oncol 2005;90:195-207.

33 Demetri GD, Benjamin RS, Blanke CD, et al. NCCN Task Force report: management of patients with gastrointestinal stromal tumor (GIST)-update of the NCCN clinical practice guidelines. J Natl Compr Canc Netw 2007;5:S1-29.

34 Moilanen J, Lassus H, Leminen A, et al. Ezrin immunoreactivity in relation to survival in serous ovarian carcinoma patients. Gynecol Oncol 2003;90:273-281.

35 Ten Heuvel SE, Hoekstra HJ, Bastiaannet E, et al. The classic prognostic factors tumor stage, tumor size, and tumor grade are the strongest predictors of outcome in synovial sarcoma: no role for SSX fusion type or ezrin expression. Appl Immunohistochem Mol Morphol 2009;17:189-195.

36 Antonescu CR, Viale A, Sarran L, et al. Gene expression in gastrointestinal stromal tumors is distinguished by KIT genotype and anatomic site. Clin Cancer Res 2004;10:3282-3290.

37 Haller F, Lobke C, Ruschhaupt M, et al. Increased KIT signalling with up-regulation of cyclin $\mathrm{D}$ correlates to accelerated proliferation and shorter disease-free survival in gastrointestinal stromal tumours (GISTs) with KIT exon 11 deletions. J Pathol 2008;216:225-235. 
38 McClatchey AI. Merlin and ERM proteins: unappreciated roles in cancer development? Nat Rev Cancer 2003;3:877-883.

39 Bernards R, Weinberg RA. A progression puzzle. Nature 2002;418:823.

40 Krishnan K, Bruce B, Hewitt S, et al. Ezrin mediates growth and survival in Ewing's sarcoma through the AKT/mTOR, but not the MAPK, signaling pathway. Clin Exp Metastasis 2006;23: $227-236$.
41 Siesser PM, Hanks SK. The signaling and biological implications of FAK overexpression in cancer. Clin Cancer Res 2006;12:3233-3237.

42 Poullet P, Gautreau A, Kadare G, et al. Ezrin interacts with focal adhesion kinase and induces its activation independently of cell-matrix adhesion. J Biol Chem 2001;276:37686-37691.

43 Monni R, Haddaoui L, Naba A, et al. Ezrin is a target for oncogenic Kit mutants in murine erythroleukemia. Blood 2008;111:3163-3172. 